\title{
Both DQA1 and DQB1 genes are implicated in HLA-associated protection from Type 1 (insulin-dependent) diabetes mellitus in a British Caucasian population
}

\author{
D.A. Cavan, K.H.Jacobs, M. A.Penny, M. A. Kelly, C.Mijovic, D. Jenkins, J. A. Fletcher and A.H. Barnett \\ Department of Medicine, University of Birmingham and East Birmingham Hospital, Birmingham, UK
}

\begin{abstract}
Summary. Inherited susceptibility to Type 1 (insulin-dependent) diabetes mellitus is partly determined by HLA genes. It has been suggested that protection from disease may be conferred by HLA-DQB1 genes which encode molecules with aspartate at position 57 . We investigated the contributions of HLA-DRB1, DQA1 and DQB1 genes to protection from disease. Restriction fragment length polymorphism and sequence specific oligonucleotide analysis in 156 British Caucasian Type 1 diabetic and 116 control subjects showed protection from disease was associated with DR2, DRw6 and DR7 haplotypes. The most protective DQA1 allele was DQA1*0102 which occurred on both DR2 and DRw6 haplotypes. The DQB1 alleles DQB1*0303, DQB1*0602 and DQB1*0603 were associated with protection, as was
\end{abstract}

DQB1*0604, which encodes an Asp- 57 negative DQ $\beta$ molecule. Heterozygosity for both protective and predisposing HLA markers was reduced in diabetic compared with control subjects. We conclude that both DQA1 and DQB1 genes are implicated in HLA-associated protection from Type 1 diabetes in this British Caucasian population. The overall structure of the DQ heterodimer is critical and DQ $\beta$-Asp 57 is of secondary importance in determining protection from disease. The effect of protective HLA types may predominate over that of predisposing markers.

Key words: Type 1 (insulin-dependent) diabetes mellitus, HLA genes, protection.
HLA genes contribute 30 to $60 \%$ of genetic susceptibility to Type 1 diabetes mellitus [1,2]. Early serological data established positive disease associations with DR3 and DR4 and a negative association with DR2 [3]. The finding of stronger disease associations with certain HLA-DQ alleles has focussed attention on the DQA1 and DQB1 genes as susceptibility determinants [4]. These encode the $\alpha$ and $\beta$ chains of DQ molecules which are expressed by antigen presenting cells, where they present antigen to CD4 + T cells.

Previous studies have shown evidence of a protective effect of HLA genes [5]. It has been suggested that HLAassociated protection is mediated by DQB1 alleles which encode a $\beta$-chain with aspartate at position 57 (Asp 57positive), possibly by affecting binding of antigen to the DQ molecule, whereas other amino acids at this position (Asp 57-negative) predispose to disease [6]. There are important arguments against this hypothesis [7] and it is likely that other factors are involved in determining protection from disease.

More recently, DQA1 alleles which encode an $\alpha$-chain with arginine at position 52 have been shown to be positively associated with disease [8]. The only common Arg 52- positive DQA1 alleles in Caucasians, however, are those occurring on DR3 and DR4 haplotypes and it is possible that the disease correlation with Arg-52 merely reflects the known predisposing effects of these haplotypes. Alternatively, DQ-mediated protection may result from an effect on the T-cell repertoire, for which there is evidence from animal studies [9].

The consistent negative disease association of certain DQB1 alleles in different races [4] suggests a direct protective effect. Alternatively the effect may be secondary to linkage disequilibrium with the DQA1 gene or other as yet unidentified genes. As both DQA1 and DQB1 genes determine the structure of the DQ molecule, it is possible that both are important in determining protection from the disease.

DQ molecules may be encoded by DQA1 and DQB1 alleles on the same haplotype (in cis position) or on different haplotypes (in trans position) [10]. Although expression of certain DQ molecules in trans has not been demonstrated, it has been suggested that particular DQ heterodimers encoded either in cis or trans may be important in determining susceptibility to Type 1 diabetes. 
The aims of the present study were to determine: 1) the DR types and DQA1 and DQB1 alleles which are associated with protection from Type 1 diabetes in a British Caucasian population, 2) whether protection is predominantly conferred by the DQA1 or DQB1 genes and 3) whether the effect of a protective marker predominates over that of a predisposing marker.

\section{Subjects and methods}

\section{Subjects}

One hundred and fifty-six subjects with Type 1 diabetes and 116 racially-matched control subjects were studied. All subjects were British Caucasians and currently resident in Birmingham or the surrounding area. They were diagnosed below 30 years of age with acute onset of symptoms and absolute dependence on insulin from the time of diagnosis. Control subjects were racially matched with no personal or family history of diabetes. Selected data concerning DR types from the diabetic subjects and 71 of the control subjects have been reported previously [11].

DNA was extracted from $30 \mathrm{ml}$ venous blood using a phenolchloroform extraction method. DR types were identified by restriction fragment length polymorphism analysis [12]. DNA $(7 \mu \mathrm{g})$ was digested with Taq I restriction enzyme to identify DR types. Bam HI digests were subsequently performed to distinguish DR3 and DRw6 and Hind III digests to distinguish DR7 and DR9. Digested fragments were separated and blotted onto nylon filters. Taq I and Hind III fragments were hybridised with radiolabelled cDNA consisting of the 500 base pair Pst fragment of pII- $\beta-4$, and Bam HI fragments were hybridised with radiolabelled cDNA consisting of the Hind III/Pst insert of pII- $\beta-1$ (full length DQB1 gene). DR2 and DRw6 subtypes were deduced from the associated DQB1 alleles. DR2 in association with DQB $1 * 0602$ and DQB $1 * 0601$, respectively were typed DRw15 (Dw2) and DRw15 (Dw12). DRw6 in association with DQB1*0603, DQB1*0604 and DQB1*0503, respectively were typed DRw13 (Dw18), DRw13 (Dw19) and DRw14 (Dw9).

\section{Sequence specific oligonucleotide typing of $D Q A 1$ and $D Q B 1$ alleles}

DQA1 and DQB1 alleles were identified using oligonucleotide probing of polymerase chain reaction (PCR) amplified DNA. The second exons of the DQA1 and DQB1 genes were amplified from $2 \mu \mathrm{g}$ genomic DNA by PCR. The DQA 1 gene was amplified as described previously [13]. The DQB1 gene was amplified for 35 cycles using the 5' GH29 (5' GAG CTG CAG GTA GTT GTG TCT GCA CAC $\left.3^{\prime}\right)$ and $3^{\prime}$ DB130 (5' AGG GAT CCC CGC AGA GGA TTT CGT GTA CC $\left.3^{\prime}\right)$ primers. Denaturation was at $94^{\circ} \mathrm{C}$ for $1 \mathrm{~min}$ ( 5 min first cycle) and annealing/extension at $60^{\circ} \mathrm{C}$ for $1 \mathrm{~min}(5 \mathrm{~min}$ final cycle). Amplified DNA $(5 \mu \mathrm{l})$ was denatured and dot blotted onto nylon filters which were hybridised with eight sequence specific oligonucleotide probes which distinguish seven DQA1 alleles, and 15 probes which distinguish 12 DQB1 alleles [14]. In a small number of cases, successful DQB1 amplification was not possible for technical reasons. The total numbers of evaluable results for each locus are shown in the results tables.

\section{Statistical analysis}

The number of subjects in each group carrying: 1) each HLA type and allele, 2) an Asp 57- or Arg 52-positive allele, 3) each deduced DQ heterodimer and 4) both protective and predisposing markers at each locus was calculated. Differences between the groups were analysed using the chi-squared test or Fishers exact probability test
Table 1. HLA DR associations with Type 1 diabetes

\begin{tabular}{lllllc}
\hline DR & $\begin{array}{l}\text { Diabetic } \\
\text { patients } \\
n=156\end{array}$ & $\begin{array}{l}\text { Control } \\
\text { subjects } \\
n=116\end{array}$ & Rank & RR $(95 \%$ CI $)$ & $p c$ \\
\hline 1 & $26(16.6)$ & $19(16.4)$ & +1 & & \\
2 & $1(0.6)$ & $26(22.4)$ & +4 & $0.03(0.01-0.14)$ & $<10^{-6}$ \\
3 & $106(67.5)$ & $39(33.6)$ & & $4.05(2.46-6.75)$ & $<10^{-6}$ \\
4 & $104(66.2)$ & $36(31.0)$ & & $4.30(2.59-7.17)$ & $<10^{-6}$ \\
5 & $11(7.0)$ & $15(12.9)$ & 0 & & $\mathrm{NS}$ \\
w6 & $4(2.5)$ & $35(30.2)$ & +3.5 & $0.07(0.02-0.18)$ & $<10^{-6}$ \\
7 & $14(8.9)$ & $29(25.0)$ & +1 & $0.29(0.15-0.59)$ & $<5 \times 10^{-3}$ \\
w8 & $4(2.5)$ & $4(3.4)$ & -1 & & $\mathrm{NS}$ \\
9 & $3(1.9)$ & $7(6.0)$ & +1 & & $\mathrm{NS}$ \\
\hline
\end{tabular}

Number (\%) of diabetic and control subjects with each DR type. Rank, Final rank (see text for explanation); RR, relative risk; $\mathrm{CI}$, confidence interval; $p c$, corrected $p$ value

Table 2. DR2 and DRw6 subtypes

\begin{tabular}{llllc}
\hline $\begin{array}{l}\text { Deduced } \\
\text { subtype }\end{array}$ & $\begin{array}{l}\text { Diabetic } \\
\text { patients } \\
n=149\end{array}$ & $\begin{array}{l}\text { Control } \\
\text { subjects } \\
n=101\end{array}$ & RR(95\% CI) & $p c$ \\
\hline DRw15 (Dw2) & $1(0.7)$ & $16(15.8)$ & $0.05(0.01-0.22)$ & $<2 \times 10^{-5}$ \\
DRw15 (Dw12) & 0 & $4(4.0)$ & & $\mathrm{NS}$ \\
DRw13 (Dw18) & 0 & $11(10.9)$ & $0.03(0.003-0.21)$ & $<3 \times 10^{-3}$ \\
DRw13 (Dw19) & $3(2.0)$ & $18(17.8)$ & $0.11(0.02-0.33)$ & $<3 \times 10^{-4}$ \\
DRw14 (Dw9) & 0 & $4(4.0)$ & & $\mathrm{NS}$ \\
\hline
\end{tabular}

Number (\%) of diabetic and control subjects with deduced DR2 and DRw6 subtypes. RR, Relative risk; CI, confidence interval; $p c$, corrected $p$ value

Table 3. HLA DQA 1 associations with Type 1 diabetes

\begin{tabular}{lllllc}
\hline DQA1 & $\begin{array}{l}\text { Diabetic } \\
\text { patients } \\
n=156\end{array}$ & $\begin{array}{l}\text { Control } \\
\text { subjects } \\
n=116\end{array}$ & Rank & RR $(95 \% \mathrm{CI})$ & $p \mathrm{c}$ \\
\hline$* 0101$ & $27(17.2)$ & $26(23.0)$ & -1 & & \\
$* 0102$ & $3(1.9)$ & $49(43.4)$ & +3.5 & $0.03(0.01-0.09)$ & $<10^{-6}$ \\
$* 0103$ & $3(1.9)$ & $11(9.7)$ & +1 & $0.21(0.06-0.66)$ & $<0.035$ \\
$* 0201$ & $12(7.6)$ & $28(24.8)$ & +1 & $0.27(0.13-0.54)$ & $<0.004$ \\
$* 0301$ & $112(71.3)$ & $42(37.2)$ & & $4.33(2.61-7.17)$ & $<10^{-6}$ \\
$* 0401$ & $4(2.5)$ & $4(3.5)$ & -1 & & $\mathrm{NS}$ \\
$* 0501$ & $113(72.0)$ & $44(38.9)$ & & $4.15(2.51-6.89)$ & $<10^{-6}$ \\
\hline
\end{tabular}

Number $(\%)$ of diabetic and control subjects with each DQA1 allele. Rank, Final rank (see text for explanation); RR, relative risk; CI, confidence interval; $p c$, corrected $p$ value

as appropriate. $P$ values were corrected $\left(p_{c}\right)$ for the number of comparisons made at each locus for HLA types and alleles. $P$ values for heterodimer frequencies were multiplied by the total number of deduced molecules. Relative risk (RR) values were calculated for markers which showed significant differences between the groups. The method of Woolf with Haldane's modification for small numbers was used [15].

Rank order analysis was used to rank the DR, DQA1 and DQB1 alleles [16]. The strongly predisposing alleles were discounted to remove the effect of an excess of these markers on the frequency of other markers at that locus. Alleles were ranked in order of frequency in both groups and the net rank deduced by subtracting the rank in the control group from that in the disease group. Subsequent rank order analysis was performed following serial removal of the marker with the highest net rank, until all markers had been assigned a final rank. A positive net rank donotes a negative disease association, and a negative net rank, a positive association. 


\section{Results}

Table 1 shows the distribution of DR types among the diabetic and control groups. DR3 and DR4 were significantly positively associated and DR2 negatively associated with disease. DRw6, however, was more frequent in the control population than DR2 and was significantly negatively associated with disease. DR7 was also significantly reduced in the diabetic group compared with the control group. No subject in our population carried DRw10. Rank order analysis of DR types after removal of the predisposing DR3 and DR4 types showed DR2 to have the highest rank (+4). Ranks for other markers are shown in Table 1. Table 2 shows the deduced DR2 and DRw6 subtypes. All DR2 haplotypes which could be deduced were DRw15 (Dw2) except for four which were DRw15 (Dw12). The one DR2-positive diabetic subject was DRw15 (Dw2). The DRw6 subtypes DRw13 (Dw18) and DRw13 (Dw19) were significantly reduced in the diabetic compared with the control group.

The numbers of subjects possessing each DQA1 are shown in Table 3. DQA $1 * 0102$ was the most protective DQA1 allele and occurred on both DR2 and DRw6 (DRw13 (Dw19)) haplotypes: it was present in 1.9\% of the diabetic group and $43.4 \%$ of control subjects. DQA1*0103, which occurred on DRw6 (DRw13 (Dw18)) haplotypes, was also significantly negatively associated with disease. The DR7 associated allele DQA1*0201 was associated with protection. This allele occurred on all but three DR7 haplotypes (two diabetic and one control) which carried DQA1*0301. Rank order analysis of the DQA1 allele frequencies after removal of the predisposing DR3 and DR4-associated alleles DQA $1 * 0501$ and DQA1*0301 showed DQA1*0102 to have the highest rank $(+3.5)$.

Table 4 shows the numbers of subjects possessing each DQB1 allele in the diabetic and control groups. DQB1*0602 was associated with DR2 and was significantly protective, as were DQB1*0603 and DQB1*0604 which occurred on DRw6 haplotypes (DRw13 (Dw18) and DRw13 (Dw19), respectively). DQB1*0303 occurred on all DR9 haplotypes and 30\% of DR7 haplotypes and was significantly protective. The remaining DR7 haplotypes carried DQB $1 * 0201$, which also occurred on all DR3 haplotypes and was positively associated with disease. Rank order analysis of the DQB1 allele frequencies after removal of the DR3 and DR4-associated predisposing alleles DQB1*0201 and DQB1*0302 showed DQB1*0603 to have the highest rank $(+1)$.

Analysis of the amino acid at position 57 of the $\mathrm{DQ} \beta$ chain encoded by the DQB1 alleles showed that Asp 57 homozygosity occurred in $3 \%$ of diabetic subjects compared with $57 \%$ of control subjects; Asp/non-Asp heterozygosity occurred in $11 \%$ and $28 \%$ and non-Asp homozygosity in $86 \%$ and $15 \%$, respectively $\left(\chi^{2}=144.6\right.$, $\left.p<10^{-6}\right)$. The corresponding data for arginine at position 52 of the $\alpha$-chain were as follows: Arg 52 homozygosity occurred in $73 \%$ of diabetic and $21 \%$ of control subjects; Arg/non-Arg heterozygosity in $25 \%$ and $47 \%$ and non-Arg homozygosity in $2 \%$ and $32 \%$, respectively $\left(\chi^{2}=86.3, p<10^{-6}\right)$.
Table 4. HLA DQB 1 associations with Type 1 diabetes

\begin{tabular}{lllllc}
\hline DQB1 & $\begin{array}{l}\text { Diabetic } \\
\text { patients } \\
n=149\end{array}$ & $\begin{array}{l}\text { Control } \\
\text { subjects } \\
n=101\end{array}$ & Rank & RR $(95 \% \mathrm{CI})$ & $p \mathrm{c}$ \\
& & & & \\
$* 0201$ & $111(74.5)$ & $39(38.6)$ & & $4.44(2.66-7.85)$ & $<10^{-6}$ \\
$* 0301$ & $22(14.8)$ & $29(28.7)$ & +0.5 & $0.42(0.23-0.91)$ & $\mathrm{NS}$ \\
$* 0302$ & $87(58.3)$ & $15(14.9)$ & & $7.63(4.18-14.58)$ & $<10^{-6}$ \\
$* 0303$ & $5(3.3)$ & $20(19.8)$ & +0.5 & $0.14(0.06-0.39)$ & $<5 \times 10^{-4}$ \\
$* 0402$ & $3(2.0)$ & $3(3.0)$ & -4.5 & & $\mathrm{NS}$ \\
$* 0501$ & $22(14.8)$ & $20(19.8)$ & -1 & & $\mathrm{NS}$ \\
$* 0503$ & 0 & $5(5.0)$ & +1 & & $\mathrm{NS}$ \\
$* 0601$ & 0 & $4(4.0)$ & 0 & & $\mathrm{NS}$ \\
$* 0602$ & $1(0.7)$ & $18(17.8)$ & +0.5 & $0.05(0.01-0.20)$ & $<10^{-5}$ \\
$* 0603$ & 0 & $11(10.9)$ & +1 & $0.03(0.003-0.21)$ & $<10^{-3}$ \\
$* 0604$ & $3(2.0)$ & $18(17.8)$ & -0.5 & $0.11(0.02-0.33)$ & $<10^{-4}$ \\
\hline
\end{tabular}

Number (\%) of diabetic and control subjects with each DOB1 allele. Rank, Final rank (see text for explanation); RR, relative risk; CI, confidence interval; $p c$, corrected $p$ value

Table 5. Frequency of diabetic and control sujects heterozygous for predisposing and protective HLA markers

\begin{tabular}{|c|c|c|c|c|}
\hline & $\begin{array}{l}\text { Diabetic } \\
\text { patients } \\
n=156\end{array}$ & $\begin{array}{l}\text { Control } \\
\text { subjects } \\
n=116\end{array}$ & $\operatorname{RR}(95 \% \mathrm{CI})$ & $p$ value \\
\hline 2/3 DR & 0 & $5(4.3)$ & & \\
\hline $2 / 4$ & $1(0.6)$ & $3(2.6)$ & & \\
\hline w6/3 & $1(0.6)$ & $6(5.2)$ & & \\
\hline w6/4 & $1(0.6)$ & $7(6.0)$ & & \\
\hline $7 / 3$ & $7(4.4)$ & $10(8.6)$ & & \\
\hline $7 / 4$ & $4(2.6)$ & $4(3.5)^{\prime}$ & & \\
\hline Total DR & $14(9.0)$ & $35(30.2)$ & $0.23(0.12-0.45)$ & $<10^{-5}$ \\
\hline $\begin{array}{l}D Q A 1^{*} \\
0102 / 0301\end{array}$ & $\begin{array}{l}n=156 \\
2(1.3)\end{array}$ & $\begin{array}{l}n=116 \\
12(10.3)\end{array}$ & $0.13(0.04-0.47)$ & $<0.03^{\mathrm{a}}$ \\
\hline $0102 / 0501$ & $1(0.6)$ & $12(10.3)$ & $0.08(0.02-0.36)$ & $<0.03^{\mathrm{a}}$ \\
\hline 0103/0301 & $1(0.6)$ & $3(2.6)$ & & \\
\hline 0103/0501 & 0 & $2(1.7)$ & & \\
\hline 0201/0301 & $4(2.6)$ & $5(4.3)$ & & \\
\hline 0201/0501 & $6(3.8)$ & $9(7.8)$ & & \\
\hline Total $D Q A 1$ & $14(9.0)$ & $43(37.1)$ & $0.17(0.09-0.33)$ & $<10^{-6}$ \\
\hline$D Q B 1^{*}$ & $n=149$ & $n=101$ & & \\
\hline 0303/0201 & $1(0.7)$ & $8(7.9)$ & & \\
\hline $0303 / 0302$ & 0 & $1(1.0)$ & & \\
\hline $0602 / 0201$ & 0 & $5(5.0)$ & & \\
\hline $0602 / 0302$ & 0 & $2(2.0)$ & & \\
\hline 0603/0201 & 0 & 0 & & \\
\hline $0603 / 0302$ & 0 & $1(1.0)$ & & \\
\hline $0604 / 0201$ & $1(0.7)$ & $6(5.9)$ & & \\
\hline $0604 / 0302$ & $1(0.7)$ & $1(1.0)$ & & \\
\hline Total $D Q B I$ & $3(2.0)$ & $24(23.8)$ & $0.08(0.03-0.24)$ & $<10^{-5}$ \\
\hline
\end{tabular}

Number (\%) of protective/predisposing heterozygotes in each group. RR, Relative risk; CI, confidence interval; ${ }^{a}$ corrected $p$ value

From the combinations of DQA1 and DQB1 alleles observed in the diabetic and control groups, 64 distinct heterodimers were deduced, either expressed in cis or trans. Both the heterodimers encoded by DQA $1 * 0102$ DQB1*0602 and DQA1*0102-DQB1*0604 were significantly associated with protection from disease (Diabetic (D) $1(0.7 \%)$ vs Control (C) $18(18 \%)$ and D $2(1.3 \%)$ vs C $13(13 \%)$ respectively, $p_{c}<6.4 \times 10^{-5}$ in each case, correction factor $=64)$. The DQA1*0102-DQB1*0201 het- 
erodimer was also protective $\left(p_{\mathrm{c}}<0.032\right)$, occurring in one diabetic and 11 control subjects. Three of these control subjects carried DR7-DQB1*0201 and eight carried DR3DQB $1 * 0201$ haplotypes. The DQA1*0103-DQB1*0603 heterodimer was reduced in diabetic subjects (D 0 vs $C 7$ $(7 \%)$ ), as was the DQA $1 * 0201-\mathrm{DQB} 1 * 0303$ heterodimer (D $3(2 \%)$ vs C $12(12 \%)$ ), although the differences were not significant following correction $\left(\chi^{2}=9.7\right.$ and 9.0 , respectively).

Table 5 shows the DR and DQ genotypes heterozygous for a protective and predisposing marker. The total number of subjects with DR genotypes heterozygous for a protective (DR2, w6 or 7) and predisposing (DR3 or 4) marker was significantly reduced in the diabetic compared with the control group (D $14(9.0 \%)$ vs C 35 $\left.(30.2 \%), p<10^{-5}\right)$. DQA1 genotypes heterozygous for a protective (DQA $1 * 0102$, DQA $1 * 0103$ or DQA $1 * 0201$ ) and predisposing (DQA $1 * 0301$ or DQA $1 * 0501$ ) allele were reduced in the diabetic group (D 14 (9\%) vs C 43 $\left.(37.1 \%), p<10^{-6}\right)$. DQB1 genotypes heterozygous for a protective (DQB1*0303, DQB1*0602, DQB $1 * 0603$ or $\mathrm{DQB} 1 * 0604$ ) and predisposing (DQB $1 * 0201$ or DQA $1 * 0302$ ) allele were also reduced in the diabetic group (D 3 (2\%) vs C $24(23.8 \%), p<10^{-5}$.

Analysis of the four deduced DR-DQ haplotypes which were significantly protective gave relative risk values similar to those of the associated DQB1 alleles (DR2-DQA1*0102-DQB1*0602, RR 0.05; DRw13 (Dw19)-DQA1*0102-DQB1*0604, RR 0.11; DRw13 (Dw18)-DQA1*0103-DQB1*0603, RR 0.04; DR7DQA1*0201-DQB1*0303, RR 0.11).

\section{Discussion}

This is the largest study of protective DR, DQA1 and DQB1 associations in a Caucasian diabetic population. We confirm the well-established negative disease association with DR2. We have demonstrated, however, that DRw6 and DR7 are also associated with protection from disease in this race.

The strength of the association with DRw6 has not been reported in previous studies and reflects the negative association of both DRw13 (Dw18) and DRw13 (Dw19) in the present study. In one large population study, DRw6 was positively associated with disease [17]. Other studies have shown DRw13 (Dw18) to be neutral [3] or weakly protective [18] and DRw13 (Dw19] predisposing [6]. There is evidence that DRw6 types are more frequent in British subjects than in some other European populations [19], but this alone would not explain the marked difference in frequency between diabetic and control subjects. Confusion between DR3 and DRw6 using older serological typing reagents may account for some of the difference between our data and earlier studies.

DQA $1 * 0102$ occurred in $43 \%$ of the control group and represented the single most frequent $D Q$ allele in this group, occurring on both DR2 and DRw6 haplotypes. This indicates that the DQA1 locus may have a role in conferring protection from disease. The only predisposing haplotype carrying DQA1*0102 is DRw16-DQA1*0102DQB1*0502 [20]. This is very rare and was not present in our study population. It is possible that the protective effect of DQA1*0102 may be modified by a strong predisposing effect of the DQB1 allele on this haplotype. The occurrence of DQA1*0102, an Arg 52-negative allele, on a predisposing haplotype argues against DQ $\alpha$ Arg 52 positivity as a primary determinant of disease susceptibility.

No single DQB1 allele occurred on both DR2 and DRw6 haplotypes: DR2 was associated with DQB1*0602 and DRw6 was associated with DQB1*0603 and DQB1*0604. All were significantly protective in this study although previous studies have shown only weak protective or neutral effects of DQB $1 * 0604[17,21]$. DQB $1 * 0602$ and DQB $1 * 0603$ are Asp-57 positive but DQB1*0604 is an Asp 57-negative allele which argues against a primary role for Asp 57 in conferring protection from disease. This is supported by our finding that the Asp 57-positive allele DQB1*0402 had a negative net rank, indicating a predisposing effect, although it was rare in our population.

Further evidence of the role of the DQA1 locus comes from the negative disease association of DR7 haplotypes, almost all of which carried DQA $1 * 0201$ which we have shown to be significantly protective. This contrasts with the negroid DR7 haplotype which carries DQA1*0301 and is positively associated with disease [22].

DQB $1 * 0303$ was significantly negatively associated with disease. It occurred on $30 \%$ of DR7 haplotypes and all DR9 haplotypes. DR9 haplotypes also carried the predisposing DQA $1 * 0301$ allele and it is possible that the opposing effects of the DQA1 and DQB1 alleles on this haplotype resulted in the neutral disease association of DR9. The same haplotype is, however, positively associated with disease in the Japanese [23]. The above findings may be explained by the presence of an unidentified predisposing gene in linkage disequilibrium with DQB $1 * 0303$ in the Japanese.

Heterodimer analysis largely confirmed the haplotype associations identified in this study. An additional finding, however, was that the heterodimer encoded by DQA $1 * 0102$ and DQB $1 * 0201$ in trans was significantly protective. This finding could arise from an excess of $\mathrm{DR} 2 / 7$ or DRw6/7 heterozygotes in the control group, which would also carry the protective DQA $1 * 0201$ allele. Of the 11 control subjects in whom this molecule could be expressed, however, only three carried DR7. The remainder carried DR3, in whom the protective effect of DQA $1 * 0102$ predominates over the predisposing effect of DQB $1 * 0201$. This emphasises how disease association may be determined by the combination of DQA1 and DQB1 alleles, irrespective of the overall disease association or Asp 57-status of any single allele. Further studies are required to demonstrate the existence of molecules expressed in trans before conclusions can be drawn about any protective effect in Type 1 diabetes.

Our data on DR and DQ genotypes heterozygous for both a protective and predisposing marker demonstrate that the effect of a protective allele often dominates over the effect of a predisposing one, whereas the reverse does 
not occur. This suggests that HLA-associated protection from disease is a true phenomenon and that the protective associations described are not merely an effect of the predominance of DR3 and DR4 haplotypes in the diabetic population. This is consistent with the rank order analysis. The relative risks of protective/predisposing genotypes suggest an increasing net protective effect away from the DRB1 locus and towards DQB1. This is supported by the absence of additional protection conferred by analysis of protective DR-DQ haplotypes, confirming previous reports that protection is more strongly associated with DQ than DR alleles $[24,25]$.

This study complements our previous studies in other races. These showed consistent protective associations of DQB1*0602 and/or DQB $1 * 0603$ and DQA $1 * 0102$ and/or DQA $1 * 0103[13,14,26-28]$. The molecules encoded by these pairs of alleles are structurally very similar, differing at only two amino acid residues [6]. It is possible that these differences are not recognised by CD $4+\mathrm{T}$ cells. The DQw6 and DQw1.18 molecules formed by DQA1*0102DQB1*0602 and DQA1*0103-DQB $1 * 0603$, respectively may, therefore, directly confer protection from disease. In contrast, no DQ allele predisposes to disease in all races studied.

In summary, the DQA1 and DQB1 alleles associated with DR2, DRw6 and DR7 are associated with protection from Type 1 diabetes in British Caucasians. These data support a recent report from Finland [22] as well as the findings in the Japanese [7] that the presence of aspartate at this position is unlikely, on its own, to be an important determinant of disease protection. Our analysis does not support a role for specific heterodimers in protection from (or susceptibility to) disease. Particular DQA1 and DQB1 alleles are associated with protection and we suggest that the overall structure of the DQ molecule, determined by the individual $\alpha$ and $\beta$ chains, is important in conferring protection from disease, rather than simply aspartate at position 57 of the $\beta$-chain (or arginine on the $\alpha$-chain). Functional studies of the effect of the HLA gene products are required to determine the importance of these genes in the development of Type 1 diabetes.

Acknowledgements. DAC is an MRC Training Fellow, KHJ is supported by the British Diabetic Association, MAP and MAK by the West Midlands Regional Health Authority, and CM by the Wellcome Trust. We gratefully acknowledge financial support from the above bodies and from Eli Lilly (UK).

\section{References}

1. Rotter JI, Landaw EM (1984) Measuring the genetic contribution of a single locus to a multilocus disease. Clin Genet 26: 529 542

2. Risch N (1987) Assessing the role of HLA-linked and unlinked determinants of disease. Am J Hum Genet 40: 1-14

3. Wolf E, Spencer KM, Cudworth AG (1983) The genetic susceptibility to type 1 (insulin-dependent) diabetes: analysis of the HLA-DR association. Diabetologia 24: 224-230

4. Nepom BS, Palmer J, Kim SJ, Jansen JA, Holbeck SL, Nepom GT (1986) Specific genomic markers for the HLA-DQ subregion discriminate between DR4 + insulin-dependent diabetes mellitus and DR4 + seropositive juvenile rheumatoid arthritis. J Exp Med 164: 345-450

5. Nepom GT (1990) A unified hypothesis for the complex genetics of HLA associations with IDDM. Diabetes 39: 1153-1157

6. Todd JA, Bell JI, McDevitt HO (1987) HLA-DQ $\beta$ gene contributes to susceptibility and resistance to insulin-dependent diabetes mellitus. Nature 329: 599-604

7. Awata T, Kuzuya T, Matsuda A et al. (1990) High frequency of aspartic acid at position 57 of HLA-DQ $\beta$-chain in Japanese IDDM patients and nondiabetic subjects. Diabetes 39: 266-269

8. Khalil I, d'Auriol L, Gobet $M$ et al. (1990) A combination of HLA-DQ $\beta$ Asp 57-negative and HLA DQ $\alpha$ Arg 52 confers susceptibility to insulin-dependent diabetes mellitus. J Clin Invest 85: 1315-1319

9. Bill J, Kanagawa O, Woodland DL, Palmer E (1989) The MHC molecule I-E is necessary but not sufficient for the clonal deletion of V $\beta$ II-bearing T cells. J Exp Med 169: 1405-1419

10. Nepom BS, Schwarz D, Palmer JP, Nepom GT (1987) Transcomplementation of HLA genes in IDDM. Diabetes 36: 114-117

11. Jenkins D, Fletcher J, Penny MA et al. (1991) DRB genotyping supports recessive inheritance of DR3-associated susceptibility to insulin-dependent diabetes mellitus. Am J Hum Genet 49: 4953

12. Fletcher J, Odugbesan O, Mijovic C, Mackay E, Bradwell AR, Barnett AH (1988) Class II HLA DNA polymorphisms in type 1 (insulin-dependent) diabetic patients of North Indian origin. Diabetologia 31: 343-350

13. Jacobs KH, Jenkins D, Mijovic CH et al. (1992) An investigation of Japanese subjects maps susceptibility to type 1 (insulin-dependent) diabetes mellitus close to the DQA1 gene. Hum Immunol 33: 24-28

14. Jenkins D, Mijovic C, Jacobs KH, Penny MA, Fletcher J, Barnett AH (1991) Allele-specific gene probing supports the DQ molecule as a determinant of inherited susceptibility to type 1 (insulin-dependent) diabetes mellitus. Diabetologia 34: 109-113

15. Mathews JD (1984) Statistical aspects of immunogenetic association with disease. In: Simons MJ, Tait BD (eds) Detection of immune-associated genetic markers of human disease. Churchill Livingstone, London pp 106-136

16. Thomson $G$ (1984) HLA DR antigens and susceptibility to insulin-dependent diabetes mellitus. Am J Hum Genet 36: 13091317

17. Thomson G, Robinson WP, Kuhner MK et al. (1988) Genetic heterogeneity, modes of inheritance and risk estimates for a joint study of Caucasians with insulin-dependent diabetes mellitus. Am J Hum Genet 43: 799-816

18. Kohonen-Corish MRJ, Serjeantson SW, Lee HK, Zimmet P (1987) Insulin-dependent diabetes mellitus: HLA-DR and -DQ genotyping in three ethnic groups. Disease Markers 5: 153-164

19. Ronningen KS, Spurkland A, Iwe T, Vartdal F, Thorsby E (1991) Distribution of HLA-DRB1, -DQA1 and -DQB1 alleles and DQA1-DQB1 genotypes among Norwegian patients with insulin-dependent diabetes mellitus. Tissue Antigens 37: 105111

20. Cohen N, Brautbar C, Font M-P et al. (1986) HLA-DR2-associated Dw subtypes correlate with RFLP clusters: most DR2 IDDM patients belong to one of these clusters. Immunogenetics 23: $84-89$

21. Reijonen H, llonen J, Knip M, Åkerblom HK (1991) HLA-DQ and absence of Asp 57 as susceptibility factors of IDDM in Finland. Diabetes 40: 1640-1644

22. Todd JA, Mijovic C, Fletcher J, Jenkins D, Bradwell AR, Barnett AH (1989) Identification of susceptibility loci for insulin-dependent diabetes mellitus by trans-racial gene mapping. Nature 338: 587-589

23. Todd JA, Fukui Y, Kitagawa T, Sasazuki T (1990) The A3 allele of the HLA-DQA1 locus is associated with susceptibility to type I diabetes in the Japanese. Proc Natl Acad Sci USA 87: 1094-1098

24. Erlich HA, Griffith RL, Bugawan TL, Aiegler R, Alper C, Eisenbarth G (1990) Implication of specific DQB1 alleles in genetic 
susceptibility and resistance by investigation of IDDM siblings with novel HLA-DQB1 allele and unusual DR2 and DR1 haplotypes. Diabetes 40: 478-481

25. Zeliszewski D, Tiercy JM, Boitard Cet al. (1991) Extensive study of DRB, DQA and DQB gene polymorphism in 23 DR2-positive, insulin-dependent diabetes mellitus patients. Hum Immunol 33: 140-147

26. Mijovic CH, Jenkins D, Jacobs KH, Penny MA, Fletcher J, Barnett AH (1991) HLA-DOA1 and -DQB1 alleles associated with genetic susceptibility to IDDM in a black population. Diabetes 40: 748-753

27. Penny MA, Jenkins D, Mijovic CH et al. (1992) Susceptibility to insulin-dependent diabetes mellitus in a Chinese population: role of HLA class II alleles. Diabetes 41: 914-919
28. Awata T, Kuzuya T, Matsuda A, Iwamoto Y, Kanazawa Y (1992) Genetic analysis of HLA class II alleles and susceptibility to type 1 (insulin-dependent) diabetes mellitus in Japanese subjects. Diabetologia 35: 419-424

Received: 10 August 1992

and in revised form: 2 November 1992

Dr. D.A. Cavan

Department of Medicine

Queen Elizabeth Hospital

Birmingham B152TH

UK 